\title{
A156 THYROID DISEASE AND VITAMIN D DEFICIENCY
}

S Kivity, N Agmon-Levin, M Zisappl, Y Shapira, V Barak, K Dankó, Z Szekanecz, M Szyper-Kravitz, P langevitz, B Gilburd, Y Shoenfeld Center for Autoimmune Diseases, Department of Medicine 'A \& C', Sheba Medical Center, Tel-Hashomer, Israel

10.1136/ard.2010.129650g

Background The role of vitamin D as an immune-modulator has been emphasised in recent years, and low levels of this hormone were observed in several autoimmune diseases. ${ }^{1}$ Vitamin D mediates its effect through binding to vitamin D receptor (VDR) and activation of VDR-responsive genes. VDRgene polymorphism was found to associate with autoimmune thyroid diseases (AITD), however studies exploring levels of vitamin $\mathrm{D}$ in patients with AITD are scarce and yield conflicting results. We therefore evaluated the levels of vitamin $\mathrm{D}$ in patients with AITD compared with non-autoimmune thyroid disease and healthy subjects.

Methods Serum vitamin D levels were measured in 50 patients with AITDs, 42 patients with non-autoimmune thyroid diseases and 98 healthy subjects using the Liaison chemiluminescent immunoassays (DiaSorin, Italy). Vitamin $\mathrm{D}$ deficiency was designated at $<10 \mathrm{ng} / \mathrm{ml}$. Patients were evaluated for anti-thyroid antibodies, thyroid function and demographic parameters.

Results The prevalence of vitamin D deficiency was significantly higher in patients with AITD compared with healthy individuals $(72 \%$ vs $30.6 \% ; \mathrm{p}<0.001)$, and in patients with Hashimoto's thyroiditis compared with patients with nonAITD $(79 \%$ vs $52 \% ; p<0.05)$. Vitamin D deficiency significantly correlated with the presence of anti-thyroid antibodies $(p=01)$ and a trend towards an association with disturbed thyroid function was also observed ( $p=0.59)$.

Conclusion Significantly low levels of vitamin D were documented in patients with AITD, and were related to the presence of anti-thyroid antibodies and disturbed thyroid function. Although further studies are required, the role of vitamin D in the pathogenesis of autoimmune thyroid diseases is suggested and supplementation should be considered.

\section{REFERENCE}

1. Kamen DL, Cooper GS, Bouali H, et al. Vitamin D deficiency in systemic lupus erythematosus. Autoimmun Rev 2006;5:114-7. 